\section{$\underset{\substack{\text { hommes } \\ \text { \& migrations }}}{ }$}

\section{Hommes \& migrations}

Revue française de référence sur les dynamiques

migratoires

1286-1287| 2010

Les migrations subsahariennes

\title{
Immigrés africains en France et au Royaume-Uni
}

Quels liens avec l'Afrique?

\section{Jacques Barou}

\section{OpenEdition}

1 Journals

\section{Édition électronique}

URL : https://journals.openedition.org/hommesmigrations/1733

DOI : 10.4000/hommesmigrations. 1733

ISSN : 2262-3353

\section{Éditeur}

Musée national de l'histoire de l'immigration

\section{Édition imprimée}

Date de publication : 1 juillet 2010

Pagination : 110-123

ISSN : 1142-852X

\section{Référence électronique}

Jacques Barou, «Immigrés africains en France et au Royaume-Uni », Hommes \& migrations [En ligne], 1286-1287 | 2010, mis en ligne le 29 mai 2013, consulté le 21 septembre 2021. URL : http:// journals.openedition.org/hommesmigrations/1733; DOI : https://doi.org/10.4000/

hommesmigrations. 1733 


\section{Immigrés africains en France et au Royaume-Uni Quels liens avec I’Afrique?}

Par Jacques Barou, CNRS, Grenoble Recherche conduite avec le concours de Sabrina Aouici, Claudine Attias-Donfut, Rémi Gallou (Cnav), Nicole Lapierre (EHESS), Martine Segalen (université de Paris X) et Joanne Cook, Louise Waite et Petra Aigner (université de Leeds)

Difficile de maintenir le contact avec le pays d'origine de ses parents

quand on est né en immigration. En France comme au Royaume-Uni, les enfants de migrants originaires d'Afrique subsaharienne entretiennent des liens multiples avec le continent africain. Leurs attitudes dépendent à la fois des représentations culturelles léguées par leurs aînés et de leur

expérience directe de cet ailleurs si proche. D'une solidarité affirmée avec un pays, une ethnie ou l'Afrique en général au refus d'un héritage déterminé par avance, entrée dans la fabrique des identités. 
Une nouvelle diaspora africaine s'est constituée en Europe au cours des dernières décennies du vingtième siècle. La France et la Grande-B retagne, qui étaient auparavant les deux principales puissances coloniales sur le continent africain -où elles ont conservé d'importantes zones d'influence après les indépendances -, sont les deux principales terres d'accueil en Europe pour une immigration qui compte aujourd'hui une seconde génération disposant de la citoyenneté européenne. Une recherche comparative concernant les populations d'origine subsaharienne de ces deux pays a été engagée en 2008 à l'initiative, côté français, de la cellule recherche de la Cnav, du CNRS et de l'université de Nanterre, et de l'université de Leeds, côté britannique. Il s'agit d'une approche résolument qualitative fondée sur des entretiens individuels et collectifs réalisés auprès de ressortissants de divers pays africains et de leurs enfants adultes, pour la plupart nés en France ou au Royaume-Uni ${ }^{(1)}$. Malgré la distance, l'espacement des allers-retours et parfois le rejet du pays chez les immigrés de première génération et malgré les fortes tendances à l'acculturation chez les jeunes, le devenir du continent africain ne laisse pas indifférent cette population, même si, dans sa grande majorité, elle semble pourtant avoir fait le choix de vivre définitivement en Europe.

Sans avoir d'ambition de représentativité statistique, les échantillons enquêtés reflètent les composantes essentielles des immigrations subsahariennes, sensiblement différentes en France de ce qu'elles sont en Grande-Bretagne, du point de vue des origines, mais pas trop éloignées du point de vue des profils sociodémographiques.

\section{Une démarche comparative}

En France, les derniers chiffres disponibles attestent de la diversification de l'immigration africaine. La population immigrée en provenance des pays d'Afrique subsaharienne comptait, selon le dernier recensement de la population ${ }^{(2)}, 570000$ personnes sur un total de 4930000 immigrés, soit 11,5\% de l'ensemble. Cette proportion reste relativement modeste par rapport à celle des immigrés d'Afrique du Nord (30,4 \%) et d'Europe (40\%).

Toutefois, c'est le nombre d'immigrés subsahariens qui a connu la plus forte augmentation en pourcentage entre les deux derniers recensements : $+45 \%$, contre $+17 \%$ pour les originaires d'Afrique du Nord. Cette forte augmentation s'inscrit dans la continuité d'une croissance régulière amorcée dès les années soixante. On observe aussi un élargissement considérable des pays de provenance. Tous les pays du continent africain sont représentés aujourd'hui en France. Ce n'est pas nouveau, car l'éventail des pays d'origine a toujours été très large. Ce qui est nouveau, par contre, 
c'est la croissance considérable de plusieurs groupes nationaux dont les effectifs étaient jusque-là assez limités, comme les natifs du Congo Brazzaville qui se placent en troisième position, juste derrière les Maliens, avec près de 40000 personnes, ou les natifs de Côte d'Ivoire qui sont passés d'un peu plus de 12000 en 1982 à plus de 30000 aujourd'hui. Cette diversité d'origine implique des diversités de trajectoires, de statuts administratifs et de conditions sociales. Le nombre de personnes venues dans le cadre du regroupement familial ou de la demande d'asile politique a fortement progressé par rapport au nombre de celles qui étaient venues dans le cadre de l'immigration économique ou de l'immigration étudiante, dominantes jusqu'aux années quatre-vingt. Cette opposition entre une immigration de travail ancienne et une immigration familiale et politique plus récente explique le paradoxe que l'on trouve au niveau des diplômes. Sur l'ensemble de la population subsaharienne âgée de 30 à 49 ans, on note à la fois un taux élevé de personnes sans diplômes (34 \%) et de diplômés du supérieur ( 27 \%). 24 \% ont un niveau BEPC, BEP ou CAP et $15 \%$ un niveau baccalauréat ${ }^{(3)}$. En ce qui concerne le travail, le taux d'activité est en moyenne plus élevé chez les Subsahariens que chez l'ensemble des immigrés : 80 \% contre 76 $\%$. Il est surtout plus élevé chez les femmes : $68 \%$ contre $62 \%$. Toutefois on observe une constante, la forte proportion d'immigrés originaires de pays qui ont été sous administration française. En 2006, ils constituent encore $70 \%$ de la population immigrée subsaharienne française.

\section{L'importance des liens historiques}

Au Royaume-Uni, le nombre d'Africains subsahariens était de 485277 selon le recensement de 2001, ce qui représentait $0,8 \%$ de la population totale du pays et 10,5\% de l'ensemble des minorités. Comme dans le cas français, la grande majorité d'entre eux vient des anciennes colonies. Les personnes nées au Nigeria forment $16 \%$ du total, celles nées au Ghana $10 \%$, celles nées en Somalie $8 \%$. Les originaires du Zimbabwe totalisent $4 \%$ et ceux originaires de l'Ouganda, de la Sierra Leone et du Kenya chacun $3 \%$. Le pourcentage de ceux qui sont nés au Royaume-Uni est très élevé : $33,7 \%$.

Au départ, les entrées ont été facilitées par l'appartenance de ces pays au Commonwealth. Mais, très vite, les règles concernant l'entrée et l'accès à la citoyenneté britannique se sont durcies et les lois actuelles se réferent aux besoins du marché du travail britannique pour accorder ou non des permis de séjour. Aujourd'hui, les ressortissants de ces pays longtemps liés à la Grande-Bretagne ne bénéficient plus d'aucun régime de faveur. 
Les raisons de l'immigration subsaharienne y sont du même ordre qu'en France, même si l'immigration de travail est plus faiblement représentée -, elle l'est essentiellement par des flux dirigés à la fin des années quarante vers les zones portuaires et industrielles et des arrivées plus récentes liées à un appel de maind'ceuvre spécialisée qui a surtout concerné des femmes venues comme infirmières. Les flux principaux sont plutôt liés aux événements politiques. On a pu constater un afflux de Soudanais pendant la seconde guerre civile qu'a connue ce pays entre 1983 et 2005. Plus récemment, le Royaume-Uni a accueilli un nombre élevé de migrants en provenance du Zimbabwe après les élections présidentielles de 2002. Les migrations étudiantes ont également été fortes. Chez certaines nationalités comme les Kenyans, c'est une tradition bien affirmée depuis longtemps.

Du point de vue de la répartition dans les diverses zones de résidence, on observe une tendance encore plus forte qu'en France à la concentration autour de la capitale : $79 \%$ des Africains vivent dans la région du Grand Londres contre 5,1 \% dans le sudest de l'Angleterre, $3 \%$ dans l'est et $2 \%$ dans le Yorkshire.

Comme en France, la population originaire d'Afrique subsaharienne est jeune. $30 \%$ ont moins de 16 ans et $2 \%$ seulement ont plus de 65 ans. Les femmes sont également plus nombreuses (53\%). La situation du point de vue de l'emploi est plus favorable qu'en France : $18 \%$ des hommes adultes africains et $16 \%$ des femmes étaient touchés par le chômage. La proportion de personnes exerçant des activités qualifiées est supérieure à celle des personnes effectuant des travaux non qualifiés (26 \% contre $23 \%$ ). Le poids des étudiants est aussi très important ( $24 \%$ de l'ensemble).

Les profils globaux des populations subsahariennes des deux pays sont donc à la fois assez proches et assez différents pour justifier une comparaison. Le poids des jeunes nés dans les deux pays d'accueil permet d'interroger les différences de représentations de l'Afrique et les divers types de liens maintenus avec elle entre les générations. Les extraits d'entretiens réalisés dans le cadre de cette recherche donnent une idée de la complexité du lien à l'Afrique, que ce soit en France ou au Royaume-Uni.

\section{La préservation des attaches avec l'ethnie ou le pays d'origine}

Le lien à l'Afrique se concrétise à travers une multitude d'activités et de projets qui vont de l'organisation de l'aide à ceux restés au pays jusqu'à la préparation de son inhumation dans la terre des ancêtres. 
La tendance dominante au sein de cette population est de mobiliser le référent national pour s'identifier. Les personnes interrogées se rattachent quasi unanimement à leur pays de naissance, qu'elles aient ou non acquis la nationalité du pays d'accueil. En France, la référence à l'ethnie d'appartenance intervient de façon plus ou moins récurrente selon les origines des personnes considérées. Les personnes d'ethnie soninké mentionnent plus facilement cette appartenance que d'autres, probablement parce qu'elles l'estiment déjà bien connue des enquêteurs mais aussi en référence à un milieu associatif bien organisé et dynamique et ayant acquis de surcroît une certaine notoriété en France au cours des dernières années. De manière générale, les originaires d'Afrique de l'Ouest font plus facilement référence à leur groupe ethnique que ceux originaires d'Afrique centrale.

L'enquête britannique fait ressortir que pour plusieurs personnes, l'identité "tribale" est plus importante que l'identité nationale. C'est sans doute là une manière d'exprimer un attachement plus profond à ses racines africaines, mais aussi peut-être un effet de regroupements résidentiels sur des bases plus étroitement ethniques que ce que l'on observe en France. "J'ai toujours pensé qu'il y avait plus de tribus à Leeds qu'à Harare", dit une enquêtée originaire du Zimbabwe. Tandis qu'un Soudanais précise son appartenance: "Vous ne pouvez pas prétendre que vous êtes britannique. Vous êtes principalement soudanais, mais vous êtes de telle ou telle tribu. Vous pensez encore à cette tribu."

\section{L'importance de l'entraide}

Pour cette population adulte, le lien à l'Afrique passe d'abord par l'entraide. La plupart des enquêtés cotisent à des caisses associatives qui ont pour finalité d'aider la famille restée au pays ou, quelquefois, d'y réaliser un projet. "Ici nous avons fait une association villageoise. Elle s'appelle 'Solidarité Psila'. On récolte de l'argent en faisant des pique-niques. On n'a pas de subventions. Chacun paye des cotisations. Sans les enfants, on n'aurait pas pu faire cette association. Ce sont eux qui nous ont expliqué comment faire. On a réussi à faire construire une case maternité au village. On voudrait faire des forages." (Homme Burkinabé, 60 ans, 5 enfants)

Là encore, les originaires d'Afrique de l'Ouest se montrent plus organisés que ceux d'Afrique centrale. Pour ces derniers, les aides sont de caractère plus informel et d'ambition plus limitée. Elles visent surtout à répondre à des situations d'urgence. Une jeune femme centrafricaine venue en France à l'adolescence explique le moyen très simple qu'elle a trouvé pour répondre aux sollicitations de sa famille au pays : "Quand il y a un courrier important à envoyer pour des papiers ou des choses comme ça, on 
va à l'aéroport et on le remet à quelqu'un qui va à Bangui. Tout le monde connaît un petit peu le système, les gens sont assez souples avec ça... On se donne des indications, on dit : 'C'est telle famille, ma sceur sera à l'aéroport'..."

Cette question des aides apportées au pays d'origine est plus rarement évoquée dans les réponses des personnes enquêtées au Royaume-Uni. Il est possible que en raison de leur appartenance à des milieux plus aisés, elles soient moins sollicitées par leurs proches au pays. Leurs pays sont par ailleurs plus riches que les pays d'origine des immigrés africains en France ou sont plongés, pour certains, dans un tel chaos qu'il est par avance décourageant de songer à y réaliser des investissements ou à y envoyer des aides qui ont peu de chances d'arriver aux destinataires. Pour autant, le maintien du lien au pays reste important à leurs yeux. Pour certains, il est avant tout un moyen de transmission de leur identité à leurs enfants.

\section{Transmettre une identité malgré la distance}

Pour cette mère kenyane interviewée au Royaume-Uni, aller au pays est l'occasion d'une leçon de vie pour les enfants. "Quand ils vont là-bas, ils réalisent beaucoup de choses. Par exemple, pour l'eau au Kenya, vous payez chaque gallon que vous utilisez, ce n'est pas bon marché. Si javais une caméra, je filmerais la tête quils font quand ils voient tout ça." Quand le retour ne se réalise pas mais qu'il est présent comme perpétuel projet, les enfants perçoivent cet objectif comme un moyen utilisé par leurs parents pour leur signifier qu'ils appartiennent bien à ce pays, comme l'exprime cette jeune femme somalie. "Mon père nous faisait croire que nous allions retourner un jour au pays, que nous ne

"Je respire par l'Afrique et je veux que mes enfants respirent par l'Afrique !'” resterions pas en Angleterre. Il croyait que la Somalie était le meilleur endroit au monde. Il voulait rentrer pour nous faire comprendre que c'était là-bas d'où nous venions et que cétait à ce pays que nous appartenions." Les images négatives que donnent d'eux-mêmes certains pays africains à leurs enfants incitent les parents à réagir afin d'éviter que le regard de leurs enfants sur le pays d'origine ne soit trop influencé par une actualité récente qui déforme le souvenir qu'ils voudraient garder et transmettre. En France, on trouve également quelques cas de parents pour lesquels le maintien du lien avec le pays d'origine est fondamental pour bien éduquer ses enfants. Ainsi, une mère togolaise, arrivée en France durant son enfance, se rend-elle dès que possible sur les terres de ses ancêtres afin de combler son "manque d'Afrique". Très 
attachée à son pays de naissance et à l'Afrique en général, elle entretient des relations quotidiennes avec ce continent : "Je respire par l'Afrique et je veux que mes enfants respirent par l'Afrique!' Elle a d'ailleurs ouvert une boutique de produits exotiques qui sert chaque soir de lieu de rencontre et de discussion aux habitués. Bien qu'elle ait passé plus de trente ans en France, elle se sent pleinement africaine, au point que sa fille, née en France d'un père camerounais, qui ne s'est rendue que très occasionnellement sur ses terres d'origine, se définit aussi comme togolaise.

\section{La vieillesse et la question du retour}

Le départ ayant été pensé, en principe, pour la durée de la vie active, beaucoup se posent la question du retour au pays pour y passer leur retraite. Les avis sont partagés sur ce sujet. Pour de nombreuses personnes interrogées, la suite "logique" de leur parcours migratoire est de rester en France. À l'heure de la retraite et du vieillissement, ceux-ci ne se voient pas retourner vivre durablement au pays d'origine.

Il arrive quelquefois, lorsque la préférence à demeurer en France est rapidement annoncée, que la suite de la réflexion et du discours nuance les propos, ou tout au moins, les expose dans leur grande complexité. Bien que le choix de la France comme lieu de vieillissement puisse paraître évident, des critères comme le climat, l'ambiance, la convivialité, le statut des personnes âgées conduisent à pencher parfois en faveur du pays d'origine comme lieu de retraite à privilégier. Face à un tel dilemme, les allers-retours réguliers durant la vieillesse se présentent alors bien souvent comme la solution la plus viable. C'est ce qu'envisage en fin de compte cette mère de famille originaire du Burkina mais née en Côte d'Ivoire, qui a par ailleurs des difficultés à situer son vrai pays : "À choisir entre la Côte d'Ivoire, le Burkina et la France, moi il n'y a pas photo, c'est la France. Je ne me vois pas vivre ailleurs qu'en France, mais jaurais pourtant bien voulu vieillir en Afrique. [...] Siça se trouve, à la retraite, je vais peut-être faire des allées et venues entre l'Afrique et ici, je ne sais pas trop."

En France, les qualités du système sanitaire peuvent encourager les migrants à rester. Pourtant, ce niveau de confort ou de sécurité ne peut pas toujours rivaliser avec la force de la tradition, l'appel du pays et des ancêtres. Vieillir dans son pays d'origine apparât alors comme la garantie, pour certains, d'être entourés. Le spectre de la maison de retraite agit comme un repoussoir. "En Afrique, il y a la famille et les amis qui s'occupent de nous, le respect. Ici, tu vas dans une maison de repos [de retraite], tu ne vois personne. Au village, les enfants te demandent ce qui se passait avant, on n'est pas seul. À la maison de repos, personne ne te demande." (Malien, 60 ans) 
Cette sollicitude de l'entourage n'est pas toujours perçue par les personnes qui ont maintenu des liens avec le pays. Dans l'enquête britannique, quelques personnes qui, pour des raisons de guerre, ont dû rester longtemps éloignées de leur pays disent ne plus s'y sentir à leur aise. "Quand je vais en Afrique je ne me sens pas chez moi.Je me sens triste parce que tous mes amis sont soit morts, soit partis au loin. Je ne vois plus les amis avec qui javais l'habitude de jouer. Je ne reconnais plus les gens." (Somalien, 57 ans)

\section{La mort ou l'ultime négociation avec la terre des ancêtres}

Parmi les individus rencontrés, quelques-uns ont parlé assez ouvertement de leur lieu d'enterrement souhaité. Mais, même à propos de ce lien ultime au pays, qui se concrétise par l'inhumation dans la terre des ancêtres, les avis sont partagés.

Pour certains, les descendants et la communauté exercent une influence considérable sur la décision retenue. Pour ce père malien, par exemple, ce qui compte c'est que sa famille - les enfants installés en France plus particulièrement - puisse venir facilement se recueillir sur sa tombe : "En France, ce sera plus facile pour ceux que je laisse de se recueillir sur ma tombe."

Pour d'autres, il est un devoir envers la communauté de reposer au pays d'origine, auprès des anciens. "Oui, je dois retourner au Mali, parce qu'il y a tout le monde là-bas. Il faut être enterré au village. [...] C'est une habitude, là où nos ancêtres sont enterrés."

Il semblerait que le choix personnel du lieu d'enterrement ne soit pas toujours respecté, car l'observance de la tradition et les aspects concrets liés au coût de l'inhumation priment sur le souhait du défunt, particulièrement lorsqu'il s'agit d'une femme. En témoigne cette mère de famille originaire du Mali : "Si je meurs en France, je veux être enterrée en France. Mais je sais que eux ne le feront pas! [...] Enterrer un corps ici, en France, coûte cher et, en plus, il n'y a pas de place. Chez nous, la femme est enterrée dans la famille de son mari, donc moi, de toutes les façons, je ne serai pas enterrée avec ma famille mais avec celle de mon mari. Alors être enterrée ici ou là-bas, pour moi, c'est pareil!'

\section{Les jeunes et le pays d'origine: une importance symbolique}

La majorité des jeunes interrogés, que ce soit en France ou au Royaume-Uni, a dans l'ensemble peu de connaissances et de contacts avec l'Afrique et n'envisage pas de s'y installer. Toutefois quelques-uns, surtout en France, témoignent d'un intérêt pour 
des questions liées à l'humanitaire et au développement qui pourrait les conduire à passer une partie de leur vie dans un pays africain.

Bien que fortement ancrés dans la société française, ils énoncent d'ailleurs très souvent un désir de s'investir dans la vie de leur pays d'origine, qu'il s'agisse de contribuer à son développement en y travaillant dans un secteur où les besoins sont importants ou qu'il s'agisse d'y créer une structure de soutien (appui à la scolarisation, association de lutte contre la pauvreté) ou encore d'animer en France une association de soutien à l'Afrique. Ainsi cette jeune Malienne exprime très bien la conscience d'un devoir envers le pays d'origine en prenant la relève des parents quand ceux-ci ne seront plus en état de s'investir dans des actions d'aide ou de développement ; "Même si on est né en France, nous, un jour ou l'autre on devra aller s'installer au Mali. Il faut construire un avenir là-bas aussi. Donc, il faut commencer dès maintenant." Dans ce cas précis, on peut considérer qu'il y a eu transmission intergénérationnelle d'un intérêt pour l'aide au pays d'origine.

\section{Un intérêt humanitaire et professionnel}

Les associations d'immigrés maliens se sont constituées en grand nombre pour mener des actions d'appui au pays d'origine. Elles ont entrepris de développer des partenariats avec des ONG spécialisées dans certains domaines. Progressivement, leur action s'est popularisée et médiatisée. Les jeunes ne sont pas indifférents à cela, même si leurs liens au pays sont plus distendus. La dynamique d'entraide enclenchée par la génération des parents ne semble pas devoir s'essouffler avec leurs enfants, tout au moins si l'on en croit le discours de la plupart des jeunes Maliens et Sénégalais rencontrés au cours de cette enquête. On ne retrouve pas la même volonté de prendre la relève chez des populations d'autres origines, ce qui ne signifie pas qu'il n'y ait pas d'autres formes d'intérêt pour le pays des ancêtres.

Quelquefois, ce sont plutôt des projets personnels, de caractère professionnel qui sont évoqués par certains jeunes nés en France, comme chez cette étudiante de parents burkinabé immigrés d'abord en Côte d'Ivoire. "J'ai très envie d'y retourner pour y travailler. J'ai postulé à deux postes de volontariat international. J'aimerais travailler avec les maires burkinabés pour les aider dans la décentralisation. Même dans un autre pays d'Afrique, jirai quand même. Mais jai placé le Burkina en tête de mes vøux devant la Côte d'Ivoire." (24 ans, étudiante en sciences politiques)

Malgré cet intérêt à travailler dans le pays d'origine des parents, on perçoit une certaine prudence par rapport à un engagement définitif. C'est ce qu'exprime bien cette étudiante en médecine qui voudrait se spécialiser dans un domaine de la santé 
pouvant être utile à son pays d'origine mais qui n'envisage pas pour autant de s'y installer de manière permanente: "Je suis en première année de médecine, et c'est surtout là-bas qu'ils ont besoin de médecins... Je me dis que si tout se passe bien et que je termine, pourquoi pas travailler six mois en France et six mois là-bas."

\section{Scepticisme et réticences vis-à-vis d'une installation permanente}

Le discours des parents sur l'éventuel "retour" de leurs enfants en terre africaine est lui aussi mitigé : si nombre d'entre eux espèrent que leurs enfants conserveront un contact avec leurs racines, ils restent plus sceptiques quant aux capacités de ces jeunes à s'adapter et s'intégrer en Afrique. Comme cette mère guinéenne qui se fonde, pour cela, sur sa propre expérience de déphasage lors de son premier retour après quelques années passées en France : "Ce qui est sûr c'est qu'on sait qu'ils ne vont pas prendre l'initiative un jour d'aller s'installer en Afrique. Ils ne pourront pas supporter la vie de là-bas. Même nous qui sommes nés et avons grandi là-bas, si on vient passer plus d'une dizaine d'années ici, on a du mal à vivre là-bas."

Les projets de retour au pays, dans un cadre professionnel ou humanitaire, sont surtout le fait de jeunes appartenant aux vieilles immigrations d'Afrique de l'Ouest, avec des parents peu instruits et faiblement qualifiés mais fortement impliqués dans des réseaux d'entraide au pays d'origine. Les

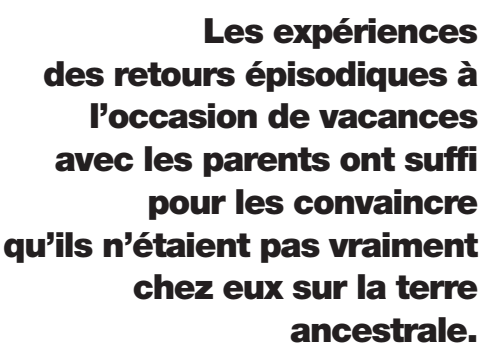
jeunes issus des immigrations plus récentes, originaires d'Afrique centrale et de milieux sociaux plus favorisés, font souvent le constat que leurs parents sont faiblement impliqués vis-à-vis de l'Afrique et disent qu'ils ne se sont guère tentés de s'y installer. C'est le cas de cette étudiante dont la mère est originaire du Cameroun. En dehors de ce lien à l'Afrique qu'elle a maintenu à travers sa mère, elle dit n'avoir aucun autre intérêt pour l'Afrique. Quitte à aller à l'étranger, elle préférerait un autre pays d'Europe plutôt que le pays de ses parents ou un autre pays africain. "Ce n'est pas que je ne vois pas l'intérêt d'y retourner, mais pour voir qui ?[...] Je sais quil y a beaucoup de choses à développer par contre, parce que ma mère m'en parle souvent. Pendant un moment, je sais qu'elle commençait à penser à faire de l'import-export. Moi, je pars du principe que j'ai fait mes études ici et que ça me servira ici." Même imprécis et timorés, les projets des jeunes nés en France contrastent avec le 
faible investissement de leurs homologues nés au Royaume-Uni. Ceux-ci se disent pourtant attachés à leur pays d'origine, mais cet attachement n'implique pas une volonté de retour ni un investissement à caractère humanitaire ou visant au développement. Les expériences des retours épisodiques à l'occasion de vacances avec les parents ont suffi pour les convaincre qu'ils n'étaient pas vraiment chez eux sur la terre ancestrale. Alors qu'ils affirment plus facilement leur appartenance à la communauté de leurs parents et l'attachement à leur culture que les jeunes nés en France, ils disent se sentir beaucoup plus mal à l'aise lors des séjours au pays d'origine et aucun d'entre eux ne fait état de projet professionnel ou humanitaire en Afrique. Ainsi, un jeune Soudanais qui se déclare par ailleurs très attaché à son identité d'origine avoue ne pas avoir apprécié le séjour qu'il a fait dans le pays de ses parents : "Pour être honnête, j'ai ressenti que le Soudan n'était pas vraiment mon pays. Je ne pouvais pas beaucoup communiquer avec les gens. La plupart du temps, je restais à la maison. Je sortais peu. On peut dire que je m'y sentais étranger."

\section{L'intérêt pour l'Afrique dans la construction d'une identité de diaspora}

Le lien à l'Afrique que vivent les jeunes générations nées en France semble être le double héritage de l'action de leurs parents en faveur du pays d'origine et d'une certaine sensibilité à l'aide au développement qui connaît une relative popularité en France. On retrouve toutefois le rôle déterminant de la transmission.

Dans le cas britannique, si on observe chez les jeunes les mêmes ambivalences identitaires et les mêmes malaises vis-à-vis du pays d'origine, l'intérêt pour l'Afrique se traduit moins par des projets à caractère humanitaire ou professionnel que par un investissement dans la vie communautaire sur les lieux de résidence.

On observe au niveau de la seconde génération une résurgence des identités ethniques qui s'affirment dans un nouvel espace diasporique. Des jeunes qui n'ont pas d'expérience directe de vie dans leur pays d'origine affirment une identité ethnique. Cela s'observe particulièrement dans la communauté somalie de Sheffield qui est en contact avec divers compatriotes vivant dans d'autres pays de l'Union européenne et qui fait tout son possible pour faciliter leur venue au Royaume-Uni. L'état de déliquescence que connait la Somalie depuis une vingtaine d'années n'incite guère à imaginer des projets pour s'y réinstaller et tout se passe comme si une patrie de substitution se constituait au sein du Yorkshire, les jeunes et les anciens se mobilisant pour aider leurs compatriotes de la diaspora plutôt qu'un pays d'origine dont on ne peut plus guère espérer quoi que ce soit. 
L'exemple somalien est assez particulier mais beaucoup de jeunes interviewés en Grande-Bretagne, tout en s'affirmant citoyens britanniques, expriment l'idée qu'ils se perçoivent différents, ne serait-ce qu'à cause de leur couleur. Cela en conduit certains à pratiquer une forme de communautarisme noir, que critique cette étudiante somalienne qui, elle, a vécu dans un environnement diversifié et ne sent pas concernée par le militantisme racial : "Quand jétais plus jeune, je ne pensais pas que jétais noire. J'étais comme ça jusqu'à ce que jaille à l'université où j’ai rencontré un groupe de Noirs qui ont décidé que jétais noire et que je devais rejoindre la communauté afrocaribéenne. Ilsétaient obsédés par leur race.Je suis allée à un de leurs meetings et jai vu quil n'y avait que des Noirs.Je n'y suis jamais retournée.J'ai trouvé que c'était ridicule.Je n'ai pas besoin d'appartenir à une minorité pour y avoir des amis noirs."

Il serait abusif d'opposer de jeunes Français d'origine africaine, bien enracinés dans leur pays de naissance tout en marquant un intérêt solidaire et humanitaire envers le pays de leur parents, à de jeunes Britanniques peu impliqués vis-à-vis de leur pays d'origine mais cherchant à retrouver leurs racines à travers la construction d'une communauté africaine en Europe. Les attitudes par rapport à l'Afrique et les sentiments identitaires sont, dans les deux pays, très diversifiés selon les individus, leurs trajectoires et leur éducation. Toutefois, le legs transgénérationnel d'une conscience de solidarité avec l'Afrique est beaucoup plus visible en France qu'au Royaume-Uni. Cela est dû incontestablement à la présence d'une population de travailleurs en provenance des zones déshéritées d'Afrique de l'Ouest, plus importante, plus visible et plus anciennement implantée en France qu'au RoyaumeUni. Au sein de cette population et même un peu au-delà, une relation étroite à l'Afrique a pu se maintenir et franchir le seuil des générations.

Ce maintien du lien avec le pays d'origine aide certainement les jeunes à se situer entre les appartenances multiples que génère le fait d'être né en immigration. Tout en sachant d'où ils viennent, ils prennent aussi conscience de ce qui les différencie de la terre et de la culture des ancêtres et, pouvant se projeter vers un pays précis avec des projets précis, ils évitent l'idéalisation des signes qui leur rappellent leurs origines et les risques d'enfermement dans une identité fantasmée.

\section{Notes}

1. Les données recueillies ont fourni la base d'un ouvrage collectif qui sera publié fin 2010 : J.Barou (dir), De l'Afrique à la France, d'une génération à l'autre, Paris, Armand Colin, collection "sociétales", 2010.

2. Ces chiffres sont ceux fournis par l'Insee en 2009. Il s'agit d'une estimation réalisée à partir des données collectées en 2004, le recensement général de la population se faisant désormais à partir d'enquêtes annuelles étalées sur cinq ans et portant sur $8 \%$ de la population des communes de plus de 10000 habitants.

3. Source : Insee, "Les immigrés en France", édition 2005, Paris, Insee, 2005, p. 95.

4. Dobbs J., Green H. and Zealey L. (éd.), Focus on Ethnicity and Religion, Basingstoke: PalgraveMacmillan, 2006. 